\title{
Development and Evolution of the Helicoidal Plane of Dental Occlusion
}

\author{
B. HOLLY SMITH \\ Museum of Anthropology, The University of Michigan, Ann Arbor, \\ Michigan 48109
}

\section{KEY WORDS Dental occlusion, Helicoidal plane, Dental attrition}

\begin{abstract}
The helicoidal plane of dental occlusion is a composite feature involving axial inclination of teeth and effects of dental attrition. Recent studies disagree on its distribution and significance in hominoid primates. The distribution, development, and functional basis of the helicoidal plane are investigated here, based on quantitative analysis of dental morphology and attrition in 667 human and 60 chimpanzee dentitions. Helicoidal planes are nearly universal in the human and chimpanzee dentitions studied. Increasing axial inclination of molars from $\mathrm{M} 1$ to $\mathrm{M} 3$ is primarily responsible for the helicoidal plane, although attrition acts to increase its expression.

In hominoids, increased molar axial tilt appears to be associated with facial shortening and dental reduction. Population and species comparisons suggest a functional relationship with cranial structure. Progressive axial tilt of molars producing a helicoidal plane is found consistently in mammals with cheek teeth positioned partly under the cranium, as in hominids, pongids, some cebids, macropodids, ursids, and sciurids. Facial shortening is an important trend in hominid evolution and axial inclination of molars might be expected to show progressive change from Australopithecus afarensis to recent Homo sapiens.
\end{abstract}

The structure, function, and development of dental occlusion are important in studies of human mastication and cranial evolution, with clinical applications in restorative dentistry. Few enough data exist on the shape of the human occlusal plane for this to be an area of ongoing investigation.

Between 1890 and 1920, various perfect geometric figures were proposed to describe the ideal arrangement of teeth in the human arch: the circular curve of Spee (1890), Bonwill's triangle (1899), and Monson's sphere (1920) are good examples. In 1925, Campbell described a form of occlusion seen on worn Australian aborigine dentitions that seemed to contradict the idea of structure and function envisioned by Monson's spherical theory. Campbell's "compound plane" of occlusion was subsequently described in many human groups with worn dentitions (see reviews in Tobias, 1980, and Osborn, 1982). Eventually, Campbell's compound plane also was named after a perfect geometric figure: the helix (Ackerman, 1953).
A helicoidal plane of occlusion has come to be regarded as a special, perhaps unique, feature of the human dentition, appearing as a result of particular evolutionary changes in the dental arcade (Hall, 1976; Tobias, 1980). Interest in this feature was heightened considerably when Tobias (1980) proposed that the presence of a helicoidal plane distinguishes members of the genus Homo from those of Australopithecus. However, recent studies have challenged this, stating that other members of the Hominoidea share this form of occlusion (Ward, 1981; Osborn, 1982; Smith, 1983b). Unresolved issues of development and significance, and contrasting statements regarding distribution of the helicoidal plane in hominoids, make this issue worthy of further investigation.

The distribution, development, and correlates of the helicoidal plane are investigated in this study, based on quantitative analyses of dental morphology and attrition in 245

Received June 7, 1984; revised August 12, 1985; accepted August 19,1985 . 
hunter-gatherers, 422 agriculturalists, and 60 wild-shot chimpanzees. This large and diverse sample makes it possible to determine the extent to which a helicoidal plane characterizes human dentitions at various stages of wear, and how humans and chimpanzees differ in this feature. The traditional explanation that the helicoidal plane is a wear pattern created by distinctive arch width relationships is tested, and development of the occlusal plane from tooth eruption to old age is investigated. Attrition and occlusion at third molars are compared between humans and chimpanzees, with particular attention given to human population differences. Finally, the evolutionary significance of this feature is addressed through a broad survey of other mammalian orders.

\section{HYPOTHESES}

The term helicoidal is used to describe dentitions in which occlusal surfaces change in slope from mesial to distal along the tooth row: In the mandible, worn first molar surfaces slope downward to the buccal, whereas third molar surfaces slope to the lingual. Maxilla and mandible show complementary curves (see illustrations in Murphy, 1964, and Hall, 1976). The resulting twist has been likened to the curve of a propeller blade (Oranje, 1934).

In most studies, the helicoidal plane is viewed primarily as a wear pattern, i.e., the result of differing locations of wear on first and third molars (Campbell, 1925; Ackerman, 1953; Murphy, 1964; Hall, 1976; Tobias, 1980; possibly Pleasure and Friedman, 1938). Campbell's original explanation, which was dominant for 50 years, is that this special wear is caused by an unusual arch width relationship found in some human groups. In standard primate occlusion, the mandibular dental arch fits inside that of the maxilla over the whole dental arch. Molar cusps that fit entirely into a basin between opposing cusps receive the most wear (mandibular buccal and maxillary lingual cusps; see Fig. 1). The human dental arcade, however, is not simple and parallel-sided. Upper and lower segments have different shapes, and at least some human groups have a markedly widened mandible at third molars. The "arcadal hypothesis" of Campbell and others (as named by Tobias, 1980) proposes that when the mandible becomes wider than the maxilla, molar cusps are forced to occlude in reversed position (an orthodontic "cross bite").

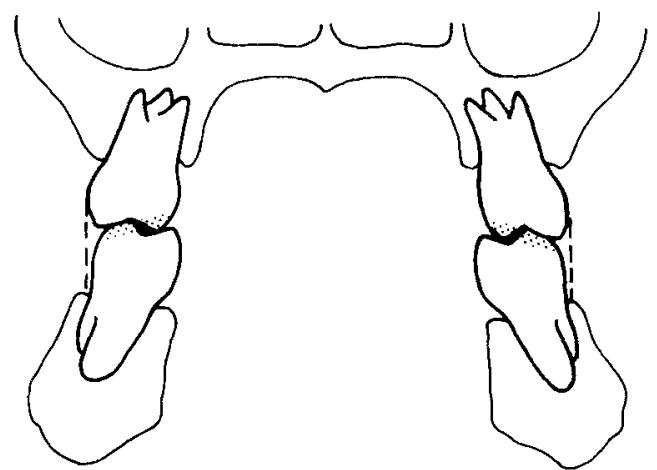

Fig. 1. A coronal section of a hominoid dental arch illustrating standard occlusion. Dashed lines emphasize the maxillary molar overjet (maxillary arch widest). The mandible fits inside the maxilla and cusps that fit into a basin between opposing cusps receive the most wear (shaded area). This configuration is thought to apply to all three molars in pongids and australopithecines. The arcadal hypothesis posits a reversal of molar occlusion at third molars in humans, where the mandible becomes wider than the maxilla.

Reversed occlusion in turn reverses the location of wear (see inset drawings in Figure 3). Since this special arch width relation occurs only at second or third molars, the worn occlusal plane takes on a twisted aspect from mesial to distal. This explanation posits a key difference in human third molar occlusion and wear as opposed to human first and second molars (and all molars of other primates). Tobias (1980) further suggested that the ultimate cause of the appearance of helicoidal planes in Homo is disproportionate maxillary dental reduction.

Some authors disagree with this emphasis on wear pattern. Drennan (1929), Van Reenen (1964), and recently Ward (1981) and Osborn (1982) note a lingual axial tilt of lower molars. A lessened degree of wear on third molars may account for maintenance of a lingually inclined surface, which begins to contrast in angle with anterior molars as wear progresses on first molars. Arch widths play no role in this explanation. Even those who emphasized wear pattern also noted that a tilted third molar axis is an important determinant of the helicoidal plane (Campbell, 1925; Ackerman, 1953; Murphy, 1964; Hall, 1976).

Finally, Moses (1946) described an association of arch widths and tooth implanation that created the helicoidal plane, with less importance ascribed to the role of dental at- 
trition. All of these studies involve testable hypotheses. The contributions of arch width, tooth implantation, and attrition to the helicoidal plane can be investigated quantitatively.

\section{MATERIALS AND METHODS}

As originally applied by Campbell, the compound (helicoidal) plane describes dentitions in which occlusal surfaces of M1 and M3 slope in opposite directions, although some authors use the term when these surfaces slope to different degrees. Strictly defined it requires that the crown of $M_{1}$ slope buccally and the crown of $\mathrm{M}_{3}$ slope lingually (in the maxilla, that $\mathrm{M}^{1}$ slope lingually and $\mathrm{M}^{3}$ slope buccally). The best way to judge the presence or absence of a helicoidal plane is to place a straight edge across the highest points of left-right tooth pairs and note the direction of slope of the occlusal surface. The direction and degree of slope can be measured with a protractor mounted on a straight edge (see figure in Smith, 1984a). In this study the deviation of the crown surface from horizontal was measured to the nearest $0.5^{\circ}$ on all maxillary and mandibular molars. Remeasurement of angles is accurate to $\pm 1^{\circ}$ in $70 \%$, and $\pm 1.5^{\circ}$ in $80 \%$ of cases. For convenience, buccal slopes are labeled positive, and lingual slopes are labeled negative. The angle measured at the occlusal surface has another useful quality; when molars are unworn, simple geometry demonstrates that the surface angle is equal to the angle of deviation of the average root axis from vertical (in an idealized symmetrical molar). Thus, the angle measured at the surface of unworn molars can be used to approximate the axis of tooth implantation (see Fig. 2).

Testing the arcadal hypothesis requires several additional measurements. Maximum arch width (at buccal surfaces of teeth) was measured at upper and lower M1 and M3. Maxillary arch width minus mandibular arch width produces one variable of interest: $\max$ illary molar overjet. A positive overjet at M3 indicates the proposed standard primate condition (mandible inside maxilla), and a negative value represents the proposed unique human condition (dominance of the mandibular arch at M3).

Each molar tooth was scored on an ordinal scale of 1-8 for amount of overall occlusal surface wear (see Smith, 1984a). This variable can serve as a measure of relative or functional age. Placement in a wear stage is

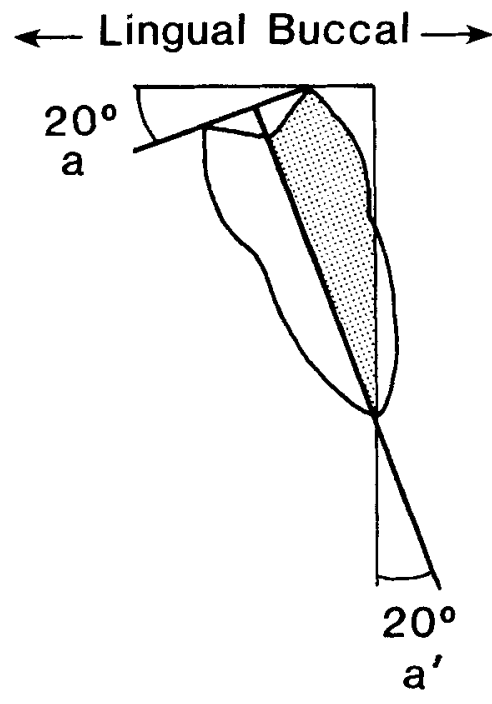

Fig. 2. Correspondence of the angle measured at the molar surface (a) to the angle of deviation of the average root axis from vertical $\left(a^{\prime}\right)$ in a coronal section of an idealized, unworn lower molar. Horizontal is defined by homologous points on right-left tooth pairs and vertical is perpendicular to this line. Deviations from symmetry caused by unequal buccal and lingual cusp heights effectively add a constant to (or subtract it from) the true axis of tooth implantation. Thus, this measure is an approximation rather than an exact measure of axial implantation. Such an approximation does not bias comparisons unless populations or species have marked and systematic differences in relative cusp height (i.e., the added factor is not a constant). Note that surface angle cannot be used to approximate axial implantation in worn teeth.

accurate in about $90 \%$ of retrials and errs \pm 1 stage for $10 \%$ of molars. Finally, one categorical variable describes the location of greatest wear on the molar crown by halves: (unworn), buccal, lingual, mesial, distal, or even over the crown surface. Critical categories of buccal and lingual wear were not mistaken in a test of 400 repeated scores.

Unless stated otherwise, results are reported for the right side, but are replaced with values from the left side if the right tooth was damaged. Given the use of both categorical and ordinal variables and some degree of asymmetry in attrition, it is preferable to use one side rather than the average of left and right values. Arch width, of course, pertains to the whole dentition.

Subjects come from ten human groups with high attrition by modern-day standards. Five hunter-gatherer groups include 27 Middle and Upper Paleolithic specimens from Europe and the Near East, 17 Mesolithic speci- 


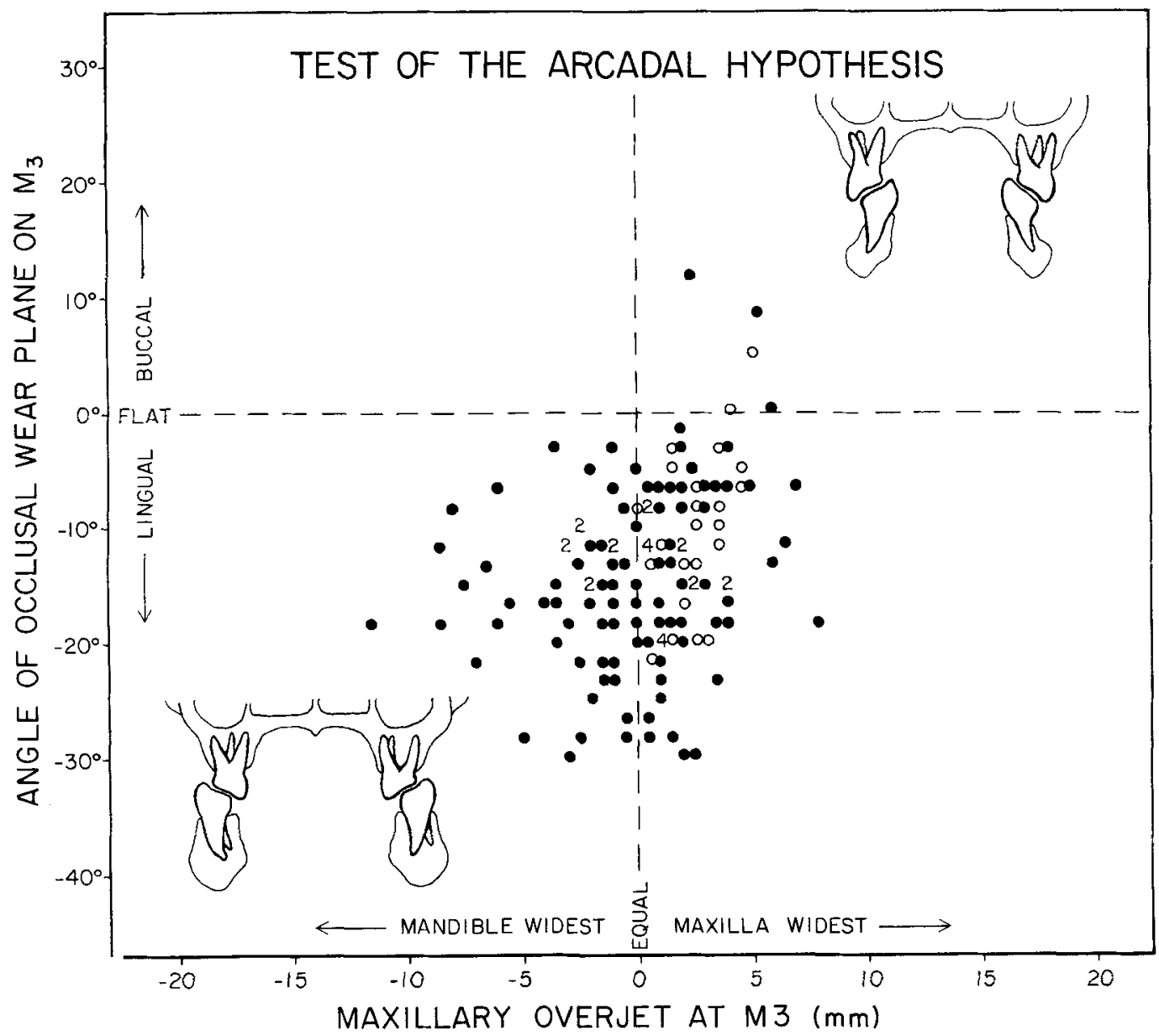

Fig. 3. A graphic test of the arcadal hypothesis for 112 humans (solid circles and digits) and 36 chimpanzees (open circles). Angle of the occlusal surface of $\mathrm{M}_{3}$ is plotted against arch width difference at M3. The zero points of a perfectly flat surface and equal arch widths are dashed in. Drawings from Tobias (1980:176) in their proper quadrants illustrate the relationships predicted

mens from coastal France, 46 Archaic American Indians from Alabama, 78 Canadian Thule and Alaskan Eskimos, and 77 Australian aborigines. Another five groups represent agriculturalists: 30 French and British Neolithic, 175 prehistoric and early historic Nubians, 105 early historic British, 57 Puebloan American Indians from New Mexico, and 55 Mississippian American Indians from Alabama. Samples are discussed in two broad subsistence divisions, since dental attrition has been shown to be similar within these divisions and different between by the arcadal hypothesis. Humans should fall in the lower left and chimpanzees in the upper right quadrant. Note that the arrangement at upper right is rare for either species. Half the humans and nearly all chimpan zees show an unexpected condition at lower right. The lingual surface angle of $\mathrm{M}_{3}$ characteristic of the helicoidal plane is not a special attribute of humans.

them (Smith, 1984a). A detailed breakdown of this sample appears in Smith (1983a, 1984a). Total available sample size is 245 hunter-gatherers and 422 agriculturalists (667 human dentitions). An additional sample of 60 wild-shot Liberian chimpanzees from the Harvard Peabody Museum are compared to humans. Sample sizes for any particular test or statistic may be much smaller depending on the tooth required, particularly for those requiring the presence of all four third molars (maxillary overjet at M3). Finally, the last section of the study includes 
results of a visual survey of 70 modern mammalian genera (primates, carnivores, rodents, insectivores, marsupials) in the collections of the University of Michigan $\mathrm{Mu}$ seum of Zoology, and Eocene representatives of these orders in the University of Michigan Museum of Paleontology.

For the purposes at hand, the 667 humans included in this study represent a broad spectrum of craniofacial form and a wide range of subsistence and diet. It is an ideal sample for determining whether the helicoidal plane is universal and investigating its development with dental attrition.

\section{RESULTS \\ Arcadal hypothesis: The role of arch widths}

A graphic test of the classic model that arch width differences control the third molar occlusal surface angle appear in Figure 3. Maxillary molar overjet (arch width difference) at third molars is on the abscissa with the zero point of equal widths indicated by a line of dashes. Occlusal surface angle of $\mathrm{M}_{3}$ is plotted on the ordinate with a dashed line drawn at $0^{\circ}$ (perfectly flat). The two conceptions of third molar occlusion described by the arcadal hypothesis are drawn in their proper quadrants. If this hypothesis is correct, chimpanzees (open circles) should fall in the upper right quadrant and Homo sapiens (solid circles) in the lower left.

These results contradict the traditional explanation encompassed in the arcadal hypothesis. The drawing in the upper right quadrant describes only a few outliers for each species. Almost all chimpanzees and about half the humans appear at the lower right-an arrangement not anticipated by the arcadal hypothesis. Despite a variety of arch width relationships, the $\mathbf{M}_{3}$ surface almost always slopes to the lingual. The within-human correlation of 0.2 (range of $0.1-0.5$ for individual human groups) and the chimpanzee correlation of 0.4 suggest that arch width difference and surface angle are related (at $P \leqslant 0.05$ ), but not in the strong, categorical fashion anticipated under the arcadal hypothesis.

The critical criterion for a helicoidal plane is a lingual slope (negative angle) on $\mathbf{M}_{3}$; points should be in the lower half of Figure 3 if this is the case. Nearly all humans and chimpanzees satisfy this requirement.

In accordance with the arcadal hypothesis, a species difference in arch widths is evident (see Table 1). At first molars, the maxillary
TABLE 1. Maxillary overjet (width of maxillary arch minus mandibular) and proportion of negative overjet compared for hunter-gatherers, agriculturalists, and chimpanzees

\begin{tabular}{lrrrr}
\hline & \multicolumn{3}{c}{$\begin{array}{c}\text { Maxillary overjet } \\
\text { (mm) }\end{array}$} & $\begin{array}{c}\text { Percentage } \\
\text { negative }\end{array}$ \\
\cline { 2 - 4 } Group & Mean & SD & $\mathrm{N}$ & \\
\hline First molar & & & & \\
$\quad$ Hunter-gatherers & 3.9 & 3.0 & 118 & 8 \\
$\quad$ Agriculturalists & 3.0 & 2.7 & 169 & 12 \\
$\quad$ Chimpanzees & 6.8 & 1.4 & 40 & 0 \\
Third molar & & & & \\
$\quad$ Hunter-gatherers & -0.4 & 3.6 & 50 & 50 \\
$\quad$ Agriculturalists & -0.1 & 3.2 & 76 & 42 \\
$\quad$ Chimpanzees & 2.5 & 1.5 & 40 & 0 \\
\hline
\end{tabular}

All three means are significantly different for $M 1$ at $P \leqslant 0.05$, and chimpanzees are different from humans for $M 3$ at $P \leqslant 0.05$.

arch is wider than the mandibular in both chimpanzee $(100 \%)$ and humans $(90 \%)$. At third molars, humans tend to have equal arch widths, with some $50 \%$ of cases showing mandibular width dominance. There are changes in maxillary overjet at M3 in both species, but these chimpanzees show no cases where the mandibular arch is widest.

\section{Molar implantation and attrition}

The occlusal surface angle of the third molar can be more easily understood by following this angle from eruption to old age. Some of the confusion regarding the helicoidal plane may stem from an idea that teeth are implanted vertically in bone and that surface angulation must be caused by differential wear. A study of 11 human skulls by Dempster et al. (1963) found that molars are implanted with an axial tilt that increased from first to third molars. These findings are illustrated in Figure 4, where root axes are projected through a human skull (redrawn from Dempster et al., 1963). This implantation produces an increasing tilt of the occlusal surfaces of unworn molars, providing a morphological basis for the helicoidal plane. Data from the present study strongly support the findings of Dempster et al. (1963). In addition, using both measurements of the occlusal surface tilt and stage of wear, it is possible to investigate the effect of wear in further developing a helicoidal plane.

The graph in Figure 5 follows the change in molar surface angle $\left(-20^{\circ}\right.$ to $\left.+30^{\circ}\right)$ from eruption to severe wear (wear stages 1-8) for mandibular molars of chimpanzee and human samples. Lines are shown from the 


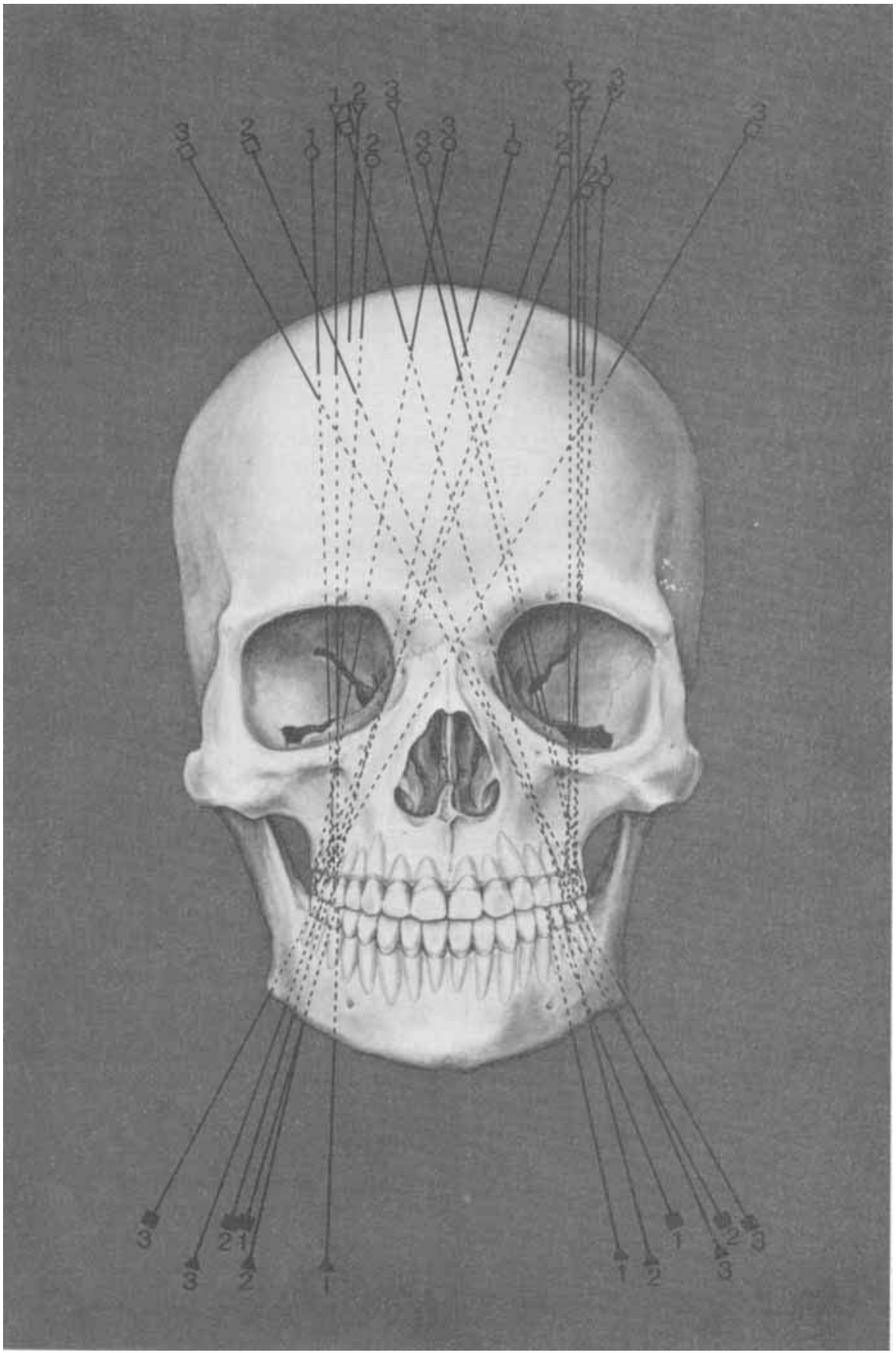




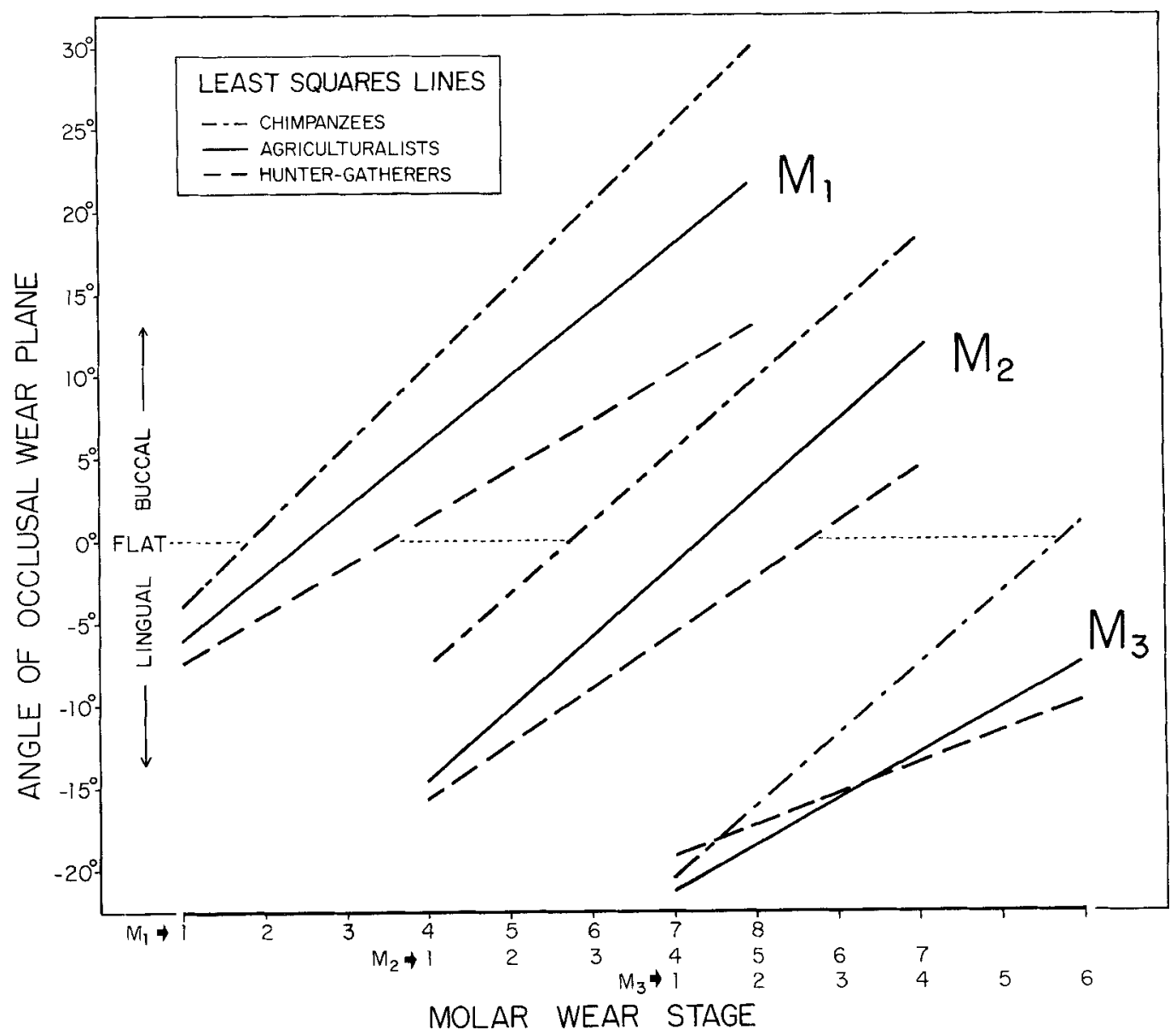

Fig. 5. Change in angle of occlusal surfaces of mandibular molars with increasing wear for chimpanzees, and human agriculturalists, and hunter-gatherers. The $\mathrm{X}$ axis is a simple stage of wear variable from 1 for unworn through 8 for teeth worn to the roots (see Smith, 1984a), with the three molars staggered in order of eruption (spread apart for clarity). All molars are plotted on the same scale of the angle of the occlusal surface $(\mathrm{Y}$ axis). These nine regression lines are based on $N=509$ at $M_{1}, N=470$ at $M_{2}$, and $N=305$ at $M_{3}$, for a total of 582 unique dentitions. All slopes differ from zero $(\mathrm{P}<0.05)$. At each tooth, all slopes differ from each other $(\mathrm{P}<0.05)$, except for human slopes at $\mathbf{M}_{3}$. Correlations of wear stage and surface angle range from 0.4 $\left(\mathrm{M}_{3}\right)$ to $0.8\left(\mathrm{M}_{1}\right)$ (Smith, 1984a). Although this graph suggests that both morphology and diet contribute to wear plane angle, the basic pattern of development is the same for both species and for all three molars. in each case a lingually oriented crown is gradually worn on the buccal half, changing the angle towards the buccal. The helicoidal plane results primarily from differential axial implantation combined with serial eruption and wear of M1 to M3.
Fig. 4. Illustration of the axial implantation of human molars, redrawn from Dempster et al. (1963). Maxillary roots are projected upwards (open symbols) and mandibular roots are projected downwards (filled symbols), with each numbered to correspond to the molar of origin (1-3). Note that the lingual tilt of mandibular molars is matched by a buccal tilt of maxillary molars and that axial tilt increases from M1 to M3. Symbols: triangles, mesial roots; squares, distal roots; circles, maxillary lingual roots. 
regression of occlusal surface angle on stage of molar wear for each tooth. Separate lines are required for chimpanzees, agriculturalists, and hunter-gatherers, for in addition to species differences in rate of change of angle, there are differences within humans attributable to diet and food preparation (see Smith, 1984a). For these purposes, however, the overall similarities are just as interesting as the differences.

Unworn molar crowns have a lingual orientation that in these humans increases from about $5^{\circ}$ at $\mathbf{M}_{1}$ to $15^{\circ}-20^{\circ}$ at $\mathbf{M}_{2}$ and $\mathbf{M}_{3}$. Chimpanzees show a similar arrangement, differing primarily by a milder axial inclination, particularly at $\mathbf{M}_{2}$. The arcadal hypothesis described a relatively horizontal $\mathbf{M}_{3}$ surface that becomes worn to the lingual. Figure 5 suggests instead that $\mathrm{M}_{3}$ is a severely tilted tooth that is worn towards the buccal. Effects of wear on surface inclination are qualitatively similar in both species and all three molars, although they differ quantitatively. The occlusal surface angle of the early-erupting first molar is rapidly changed to the buccal, but the later-erupting third molars are so severely inclined that they remain lingual until extreme old age. Molars in a single dentition are out of phase in development, and the long interval between eruption of M1 and M3 may make the helicoidal plane particularly pronounced in humans.

It may be evident that it can be misleading to compare populations by tabulating individuals with and without helicoidal planes. A strict helicoidal plane is present as soon as there is enough tooth wear to reverse the surface angle of $\mathbf{M}_{1}$ to the buccal and it endures until the entire dentition is worn out and $\mathrm{M}_{3}$ finally reverses its direction. Only the oldest members of populations with highest rates of attrition show this final stage (this is in fact the explanation for the few outliers seen previously in Figure 3). Whether a strict helicoidal plane is present thus depends on rate of wear and the age structure of the sample. Clearly, the development of a helicoidal plane is typical in the presence of moderate tooth wear in both chimpanzees and humans.

It is possible to estimate, in a general way, the contribution of morphology versus wear to the contrast in $M_{1}$ and $M_{3}$ surface angles perceived as the helicoidal plane. Expression of the helicoidal plane can be described as the difference between $M_{1}$ and $M_{3}$ surface angles. Combining information from 54 unworn $M_{1}$ 's and 59 unworn $M_{3}$ 's, it appears that axial implantation creates an initial contrast of approximately $12-14^{\circ}$ in humans (about $14^{\circ}$ in the few available unworn teeth of chimpanzees). This would be the extent of the helicoidal plane in humans in the absence of wear. The additional effect of attrition can be seen in Table 2, where this contrast is summarized for the total sample of adult individuals. Contrasts in $\mathrm{M}_{1}$ and $\mathrm{M}_{3}$ range from $0^{\circ}$ to $50^{\circ}$, but contrasts of $20^{\circ}$ are typical. Wear does apparently increase the expression of the helicoidal plane, as evidenced also by the correlation with increasing $M_{1}$ wear or "age," yet the initial morphological contribution of $12-14^{\circ}$ makes up a large part of adult values of $20^{\circ}$.

Certainly it would be possible to make finer comparisons of human populations than the broad one presented here. The effect of different modes of diet and food preparation on tooth wear patterns complicates further interpretation at this point. However, there is one further point that should be made from Table 2. These hunter-gatherers differ from agriculturalists primarily by their flatter, more even molar wear (Smith, 1984a), a pattern of wear that also tends to produce more mildly expressed helicoidal planes. This dietary association may be responsible for the reported lack of helicoidal planes in australopithecines (Tobias, 1980), which are said to show very flat wear (Clark, 1955). It is likely that helicoidal planes are present in australopithecines (see Osborn, 1982), but possibly in an exceedingly mild form.

\section{Occlusion and attrition at third molars}

The arcadal hypothesis suggested that human third molars wear in a distinctly different location (lingually on $\mathbf{M}_{3}$ ). Evidence from the present study suggests that third molars wear in a slightly different manner. This is

TABLE 2. Expression of the helicoidal plane for huntergathers, agriculturalists, and chimpanzees

\begin{tabular}{lccrc}
\hline & \multicolumn{2}{c}{ Expression (degrees) } & \\
\cline { 2 - 3 } Group & Mean & SD & N & $\begin{array}{c}\text { Correlation } \\
\text { with age }\end{array}$ \\
\hline Hunter-gathers & 18.7 & 7.3 & 70 & 0.09 \\
Agriculturalists & 22.9 & 9.6 & 122 & $0.25^{2}$ \\
Chimpanzees & 21.7 & 7.8 & 43 & $0.47^{2}$ \\
\hline
\end{tabular}

${ }^{1}$ Expression or contrast calculated as $\mathrm{M}_{3}$ angle subtracted from $\mathrm{M}_{\mathrm{I}}$ angle, using the right side only.

${ }^{2}$ Correlation with "age" $\left(M_{1}\right.$ wear stage) is significantly different from zero at $\mathrm{P} \leqslant 0.05$. 
TABLE 3. Frequency of location of greatest wear (buccal versus even) on mandibular molars of huntergathers, agriculturalists, and chimpanzees

\begin{tabular}{|c|c|c|c|c|c|c|c|c|c|}
\hline \multirow[b]{3}{*}{ Group } & \multicolumn{9}{|c|}{ Location of greatest wear } \\
\hline & \multicolumn{3}{|c|}{ M1 } & \multicolumn{3}{|c|}{$\mathrm{M} 2$} & \multicolumn{3}{|c|}{ M3 } \\
\hline & Buccal & Even & $\mathrm{N}$ & Buccal & Even & $\mathbf{N}$ & Buccal & Even & $\mathrm{N}$ \\
\hline Hunter-gatherers & 0.85 & 0.15 & 181 & 0.63 & 0.37 & 145 & 0.19 & 0.81 & 84 \\
\hline Agriculturalists & 0.99 & 0.01 & 296 & 0.73 & 0.27 & 278 & 0.23 & 0.77 & 151 \\
\hline Chimpanzees & 1.00 & 0.00 & 56 & 0.98 & 0.02 & 53 & 0.85 & 0.15 & 40 \\
\hline
\end{tabular}

Location of wear differs significantly at $\mathbf{P}<0.02$ for each tooth (within group) and for each group (within tooth position) by chi-squared tests. Location of wear is independent of stage of wear.

shown by an alteration of wear pattern visible in a comparison of the location of greatest wear on first, second, and third mandibular molars (Table 3). Of a total of 1,282 right-side mandibular molars with some wear, only two categories of location of greatest wear are common: buccal $(62 \%)$ and even $(27 \%)$. The remaining rare categories were mesial $(6 \%)$, distal $(3 \%)$, and lingual $(2 \%)$. Table 3 omits the rare categories and contrasts only buccal and even wear at each molar tooth. The table shows a remarkably clear double trend by tooth and by group. For each tooth, huntergatherers have the highest frequency of even (flat) wear and chimpanzees have the least. In each group, the frequency of even wear increases from $M_{1}$ to $M_{2}$ and $M_{3}$.

These results make several important points. The expected primate pattern of buccal wear on mandibular molars is present (certainly lingual wear is very rare), but it is not invariant for all molars in either humans or chimpanzees. Human wear patterns differ most markedly from those of chimpanzees at third molars, where even wear becomes the dominant pattern in humans. Thus there is indeed a subtle helicoidal wear pattern, most visible in humans, as suggested by Murphy (1964) and Hall (1976), although not of the categorical nature (buccal at $\mathbf{M}_{1}$ to lingual at $\mathrm{M}_{3}$ ) described by Tobias (1980). Even though third molars show some difference, these results do not suggest that normal occlusion is reversed or markedly altered.

Figure 6 shows a posterior view of human occlusion. The upper and lower arch widths are about equal at M3, yet mandibular cusps still fit inside maxillary cusps because of the axial implantation of these teeth. The wear pattern found above suggests that the occlusion and normal function of M3 is preserved (i.e., wear is not altered to the lingual), but that the extremely tilted axis and/or widened mandible at M3 contribute to wear that is more equally distributed across the crown. Although the question of which is cause and which effect (and whether advantageous or disadvantageous) remains open, it seems clear that mandibular arch width dominance must be associated with extreme axial tilting if there is to be occlusion of upper and lower third molars. If the confounding effect of age (stage of wear) is held constant in multiple regression, within-population correlations of M3 overjet and occlusal surface angle of $\mathrm{M}_{3}$ rise slightly, from $0.1-0.5$ to $0.2-0.6$, most at statistically significant levels. Thus, individuals with highly negative overjet tend to show more negatively angled third molar surfaces, with a low to moderate degree of association.

\section{Population and species differences}

In addition to within-population associations, there appear to be meaningful between-population and species differences in molar axial inclination, arch width, and facial shape. One of the greatest differences between human and chimpanzee skulls is facial prognathism. Compared to fossil pongids, hominids moved the dentition under the skull and changed the shape of the dental arcade from long and U-shaped to short and curved. It appears that molar axial inclination and arch width relationships are altered in the presence of such facial shortening.

Three human groups were chosen a priori from those with large sample sizes as examples of prognathic (Australian aborigines, Nubians) or orthognathic (Eskimos) human groups. Chimpanzees, of course, represent an extreme of prognathism outside the human range. As shown in Table 4, four dental/craniofacial variables show trends corresponding with this spectrum of facial shortening: axial inclination of $\mathrm{M}_{2}$, axial inclination of $\mathbf{M}_{3}$, maxillary overjet at $\mathrm{M3}$, and dental agenesis. Only unworn teeth were used to esti- 


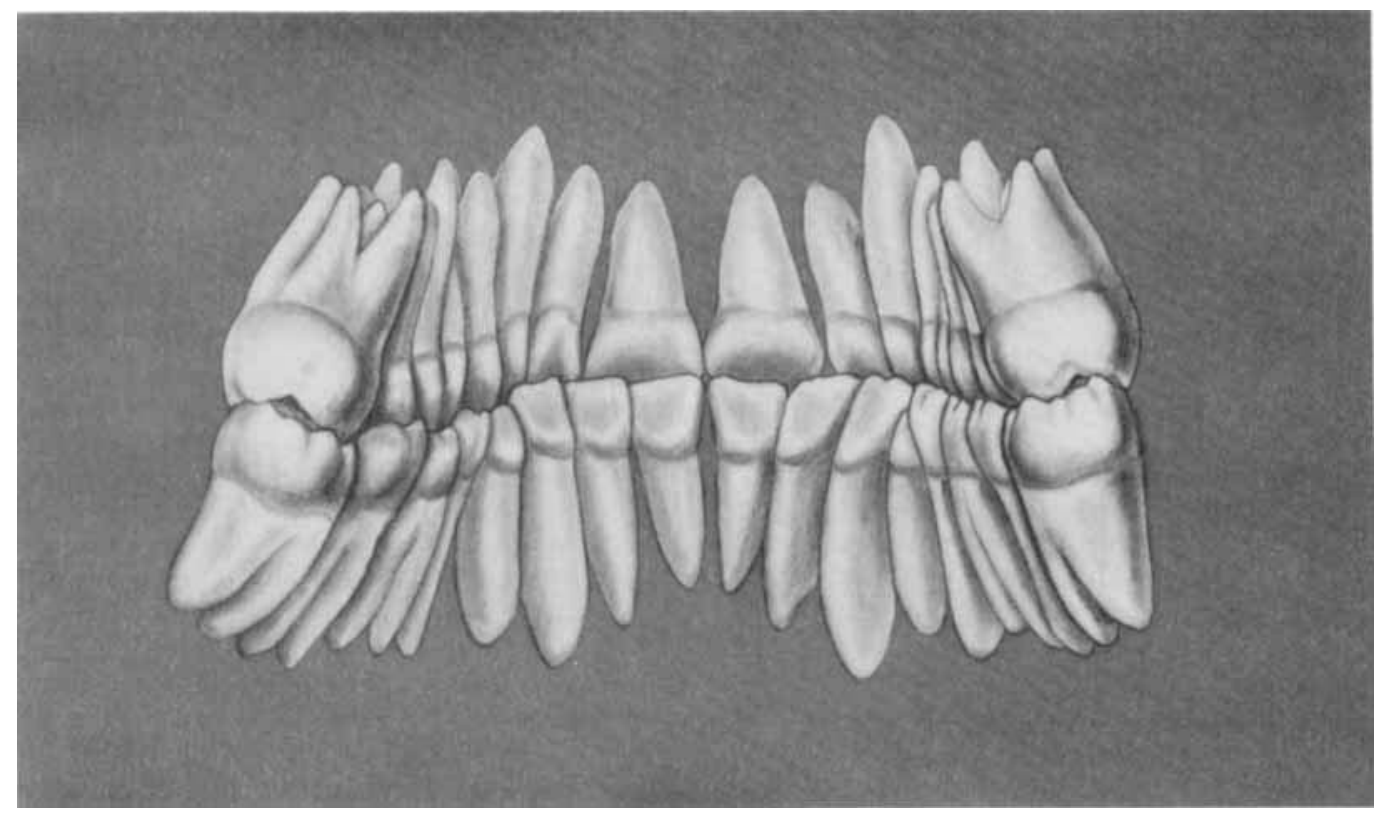

Fig. 6. A distal view of human occlusion drawn from an actual dentition with the aid of illustrations in Dempster et al. (1963). The slight asymmetry of the individual has not been corrected. Upper and lower arch widths are about equal, but mandibular buccal cusps still manage to occlude inside maxillary cusps. By rotating the axis of $\mathrm{M}_{3} / \mathrm{M}^{3}$, a wider mandible can be produced without altering cusp occlusion. Thus, relative mandibular width and axial implantation should be related.

TABLE 4. Trends in occlusal variables paralleling a spectrum of facial shortening from chimpanzees to Eskimos

\begin{tabular}{|c|c|c|c|c|c|c|c|c|c|}
\hline \multirow[b]{2}{*}{ Variable } & \multicolumn{2}{|c|}{ Chimpanzees } & \multicolumn{2}{|c|}{ Australian } & \multicolumn{2}{|c|}{ Nubian } & \multicolumn{2}{|c|}{ Eskimo } & \multirow{2}{*}{$\underset{\text { values }}{P}$} \\
\hline & $\overline{\text { Mean }}$ & $\mathrm{N}$ & Mean & $\mathbf{N}$ & Mean & $\mathbf{N}$ & Mean & $\mathrm{N}$ & \\
\hline $\begin{array}{l}\text { Axial inclination of } \mathrm{M}_{2}\left(^{\circ}\right) \\
\text { Axial inclination of } \mathrm{M}_{3}\left({ }^{\circ}\right) \\
\text { Maxillary averjet at } \mathrm{M} 3(\mathrm{~mm}) \\
\text { Dentitions with agenesis }(\%)\end{array}$ & $\begin{array}{r}-2.7 \\
-17.1 \\
2.5 \\
0.0\end{array}$ & $\begin{array}{r}3 \\
10 \\
40 \\
60\end{array}$ & $\begin{array}{r}-13.8 \\
-17.3 \\
0.8 \\
7.0\end{array}$ & $\begin{array}{r}6 \\
10 \\
26 \\
75\end{array}$ & $\begin{array}{r}-15.6 \\
-18.8 \\
0.9 \\
12.0\end{array}$ & $\begin{array}{r}6 \\
13 \\
44 \\
171\end{array}$ & $\begin{array}{r}-24.0 \\
-21.9 \\
-2.6 \\
20.0\end{array}$ & $\begin{array}{r}3 \\
4 \\
13 \\
77\end{array}$ & $\begin{array}{l}0.005^{1} \\
0.573^{2} \\
0.001^{1} \\
0.001^{3}\end{array}$ \\
\hline
\end{tabular}

${ }^{1}$ All four groups significantly different by analysis of variance. Humans differ from each other at $P \leqslant 0.05$.

2Total sample size increases from 37 to 95 if both wear stages 1 and 2 (unworn and slightly worn teeth) are used to approximate axis, giving values, respectively, of $-13.6^{\circ},-16.5^{\circ},-18.1^{\circ}$, and $-20.7^{\circ}$ that differ at $P \leqslant 0.02$.

givenesis and group are associated in chi-squared test for independence. Agenesis is computed in a conservative fashion as dentitions with agenesis relative to the total, rather than to the total known to have all 32 teeth.

mate axial implantation, so sample size is quite small (too small to include inclination of $\mathrm{M}_{1}$ ). Axial inclination of both posterior molars increases from chimpanzees through Australian aborigines, Nubians, and Eskimos. The Eskimo figure for $\mathrm{M}_{3}$ is probably an underestimate since measurements could not be taken on very short arches where third molars are lingual to the ramus. The trend in increasing axial angle of $\mathbf{M}_{3}$ is slight enough that it cannot be statistically substantiated at these sample sizes (although significant differences can be shown if sample size is increased by using both unworn and slightly worn teeth). Marked species and population differences are evident at $\mathbf{M}_{2}$, where chimpanzees show an axial inclination of only $-2.7^{\circ}$, at least $10^{\circ}$ from the nearest human value. Within humans, another $10^{\circ}$ difference separates prognathic and orthognathic groups.

Individual population figures for arch width difference at M3 also follow this trend. Whereas chimpanzees typically show a 2.5 - 
$\mathrm{mm}$ overjet (with no negative values), Australians and Nubians have values just over 0 $\mathrm{mm}$. Only in Eskimos is it typical for the mandibular arch to be widest, shown by the mean of $-2.6 \mathrm{~mm}$. Finally, Table 4 gives the proportion of dentitions with some agenesis, commonly M3, but also P4 or I2. Although not based on radiographic examination, these figures should be reasonably accurate relative to each other. Agenesis increases from essentially zero in chimpanzees to one-fifth of Eskimo dentitions. These agenesis figures are no surprise to those familiar with the human dentition, but are reported here to suggest a tie to other factors. The results taken together suggest that facial shortening may have major ramifications for the dentition.

\section{Primates and other mammals}

The association of axial inclination and facial shortening is shown for only four cases, and could be spurious rather than evidence of a functional relationship. If so, this association might fall apart in a broader comparison of primates and other mammals.

Twenty-eight mammalian families were surveyed from the orders Primates, Insectivora, Carnivora, Rodentia, and Marsupialia. Seventy genera were examined in all. Because many of these mammals have unfused symphyses (mandibles separated in dried skull material), attention was given to the axial inclination of maxillary molars. Mandibular and maxillary molars must correspond in axial inclination to some degree to occlude (see Fig. 6); in hominoids, upper and lower molar axes (surfaces of unworn molars) correspond at about $r=0.7(\mathrm{M} 1), \mathrm{r}=0.5$ (M2), and $\mathbf{r}=0.3$ (M3), and always show a complementary direction of implantation.

A visual inspection to determine the direction of axial inclination of a tooth (not worn crown surface) provided a broad and preliminary survey. Molar orientation appeared to conform to one of three major patterns shown in Figure 7, where examples from three orders are ordered into columns representing these patterns. Patterns of axial inclination of molar teeth are not randomly distributed in mammals; single arrangements often characterized whole families and no eutherian order could be found containing examples of all three possibilities. In Figure 7, the animals at right have fairly uniform molar axes, all slightly lingual. At center are animals that either have or quickly develop hel- icoidal planes because the molar surfaces already twist from mesial to distal. At left is an animal with all molar axes strongly inclined to the buccal.

The feature that unites these examples is the position of the cheek teeth relative to the junction of the zygomatic arch and maxilla (shown with a dashed line in each case in Fig. 7). In general, teeth posterior to the zygomatic root (within or medial to the temporal fossa) are strongly angled outwards to the buccal. At right, both Nasua and Alouatta show the entire molar row anterior to the zygomatic arch and a simple, mildly lingual orientation of all molars. Ursus, Cebus, and Sciurus have dentitions that cross the zygomatic arch and molars that progressively increase in axial inclination from mesial to distal. Finally, Erethizon (and many other rodents) shows the entire cheek tooth row posterior to this point and all teeth have a distinct buccal orientation. In hominoids, chimpanzees are most like Cebus at center, although humans approach the condition seen in Sciurus and some rodents.

The coherent results of this survey suggest that axial inclination of molar teeth is a biomechanical feature related to the position of cheek teeth relative to the cranium. The general rule that maxillary teeth posterior to the zygomatic root are buccally oriented also appears to apply to the more omnivorous of the Insectivora, and even to the primitive orders Multituberculata and Marsupialia, in which helicoidal planes can be found. Both multituberculates and bears create a helicoidal plane by a change in the axis of a single, elongated tooth that crosses the zygomatic arch. Marsupials include families with simple lingual inclinations, such as didelphids (opossums) and dasyurids ("native cats"), and families with helicoidal planes, such as macropodids (kangaroos and wallabies) and some phalangerids (especially Phalanger and Petaurus). The Vombatidae (wombats) approach the condition seen in eutherian rodents. An alternative to a complete axis shift seems to be a sharply incurved arcade where the palate crosses the zygomatic arch; this is found in combination with highly mesiodistally reduced last molars in some insectivores (soricids) and carnivores (canids and mustelids). Other alternatives may exist in genera not surveyed, and additional variables, such as alveolar height, probably contribute to observed variance. Although these preliminary results are presented in a cate- 
AXIAL INCLINATION OF MAXILLARY MOLARS

\section{Buccal Lingual/Buccal Lingual}

\section{CARNIVORES}

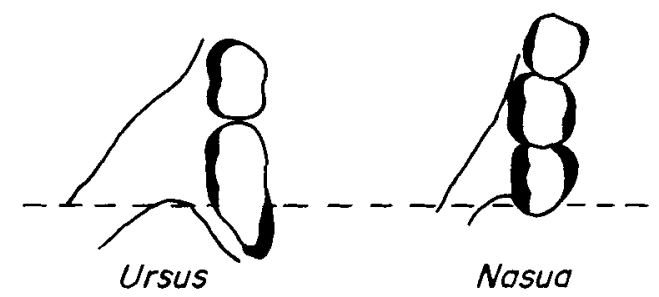

PRIMATES
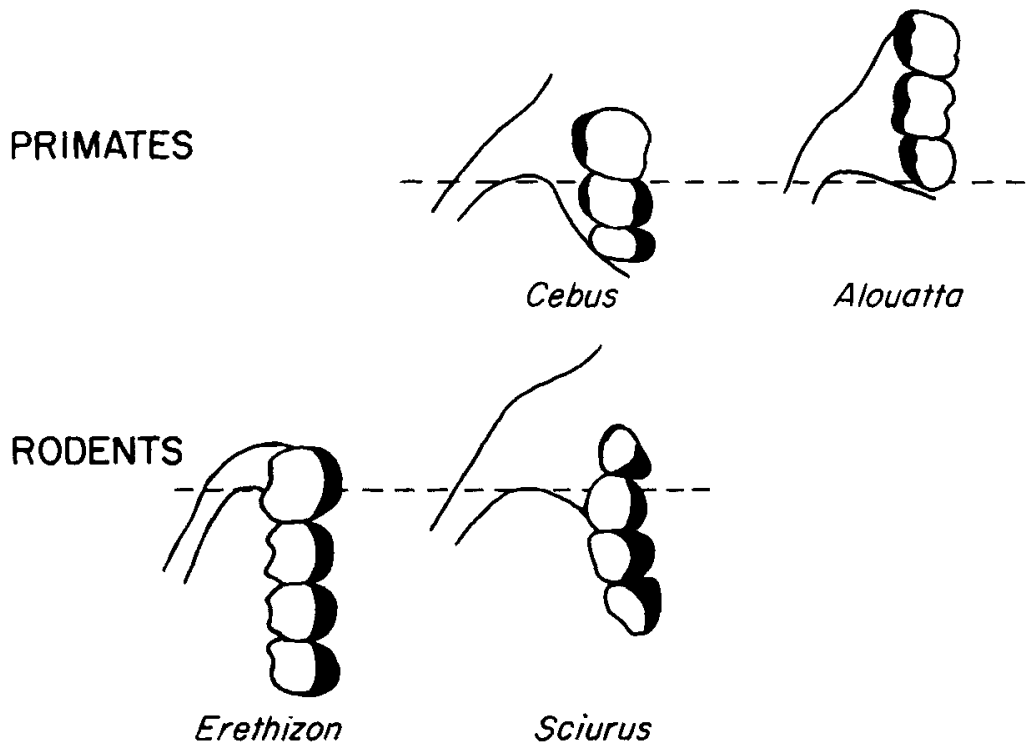

Fig. 7. Three patterns of axial implantation of molars shown for examples from the orders Carnivora, Primates, and Rodentia. Diagrams of occlusal views of the right maxilla are shown in each case with mesial at top. Sides visible in occlusal view are darkened to emphasize axial orientation. At right, Nasua (the coati) and Alouatta have simple lingual orientations of maxillary molars. At center, Ursus (bear), Cebus, and Sciurus (squirrel) show change in axial orientation from lingual to buccal; all thus have helicoidal planes. At left, Erethizon (Amer-

gorical fashion, it would be expected that detailed study would find a continuum of molar implantation and cheek tooth position.

\section{DISCUSSION AND CONCLUSIONS}

These investigations can begin to clear up some of the problems concerning the helicoidal plane-in telegraphic fashion, who, how, and why? A helicoidal plane is nearly universal in humans in the presence of moderate ican porcupine) shows all molars with a buccal orientation. The dashed line marks the juncture of the zygomatic arch with the maxilla in each case. In general, teeth posterior to the zygomatic root (lateral to the temporal fossa) show a buccal orientation of molar axes. In investigation of 70 genera, no mammal showed the hypothetical opposite condition to the helicoidal plane (buccal anterior orientation shifting to lingual at posterior molars).

tooth wear, but it also characterizes chimpanzees and a variety of other mammals including Cebus, squirrels, bears, kangaroos, and some multituberculates.

Both morphology and wear produce the helicoidal occlusal plane: a structural helicoid (of $\simeq 14^{\circ}$ contrast) is increased by normal wear (to $20^{\circ}$ or more) in both chimpanzees and humans. Hominoid molar teeth are progressively tilted and tooth wear is 
also progressively altered from $\mathrm{M} 1$ to $\mathrm{M} 3$ (concentrated more on buccal cusps at $\mathrm{M}_{1}$ and more evenly across the crown at $\mathrm{M}_{3}$ ). In humans the difference at M3 might be said to constitute a helicoidal wear pattern, but this changing pattern of wear is subtle and does not extend to a major alteration of the primate pattern of tooth wear.

Some recent humans show increased expression of the helicoidal plane due both to axial tilt of molars and to oblique (buccally restricted) molar wear, but all hominids and pongids should show this feature to some degree (Ward, 1981; Osborn, 1982). It is possible that low axial inclinations of molars (associated with facial prognathism and a deep alveolar process) and extremely flat wear (associated with diet) combine to make the occlusal plane of Australopithecus the simplest in form of all hominids (Tobias, 1980).

The arcadal hypothesis may be correct in its identification of an association of relatively expanded mandibular width, altered third molar wear, dental reduction, and pronounced helicoidal planes-features that may be increasingly expressed in more recent Homo sapiens (Campbell, 1925; Hall, 1976; Tobias, 1980). Objections to this hypothesis are that occlusion is apparently not reversed at M3 and that attrition may play a lesser role than that ascribed to it in some treatments. However, the present study must agree with statements made in many previous studies of the helicoidal plane, and sug. gests that integration of previous models is necessary rather than rejection of individual sources.

A comparison of chimpanzees with prognathic and orthognathic humans suggests that facial shortening may involve alterations of tooth axes, relative arch widths, and dental reduction. Agenesis may be simply another reflection of facial shortening, but it is possible that there is some more direct connection between extremes of axial tilting and agenesis. Molars could become nonfunctional (or have abnormal function) when axial tilting becomes extreme; it does appear that groups with the most severe axial inclination of third molars are those that are eliminating these teeth.

A survey of axial orientation of molars in several mammalian orders suggests that there is a general explanation for this feature. Axial tilting comparable to that in humans appears to be present generally in mammals that have cheek teeth that are positioned partly under the cranium and, in particular, teeth that are posterior to the $z y$. gomatic root and/or masseter insertion. The probable primitive eutherian position of the dentition relative to the cranium can be seen in Eocene mammals. Eocene carnivores, artiodactyls, and primates have long faces or rostra with the dentition separated from the cranium. Molar axes tend to be simple and slight, oriented lingually in the maxilla and buccally in the mandible. Most artiodactyls have maintained or increased the long rostrum with the dentition well separated from the cranium and have maintained this simple molar arrangement. In contrast, some carnivores, primates, and rodents have moved the cheek dentition under the cranium, and apparently with this have reversed the direction of molar implantation. Rodents appear to have accomplished this at an early date, by the time of their first appearance in North American Eocene deposits.

Buccal axial inclination of maxillary molars may simply be a way of providing support for teeth that otherwise must project roots into the temporal fossa. Alternatively, change in axial orientation may reflect an alignment of teeth with the changing line of action of masticatory muscles-a change that is more important when cheek teeth are medial to the masseter and pterygoid muscles than when they are far anterior to them. Expanded mandibular width may well be a secondary factor that is modified to preserve standard occlusion with severely tilted maxillary molars. In this view, rather than an adaptation for efficient food shearing or stable occlusion (Osborn, 1982; Hall, 1976), the helicoidal plane represents a compromise in the presence of other constraints (e.g., the need for powerful use of masseter/pterygoid given constraints of palatal and cranial architecture).

Functional explanations of severe axial tilting might also consider preliminary evidence that humans and chimpanzees avoid chewing on third molars (Smith, 1984b) and that severe axial tilting appears to be associated with third molar agenesis. Animals with all cheek teeth positioned medial to the temporal fossa appear in very few living mammalian orders-most notably in rodents and lagomorphs. Certainly these mammals possess highly modified cranial architecture and muscles of mastication. Study of the relative position of the cheek teeth, the bite point, 
and the axial implantation of molars could contribute to our understanding of mammalian jaw mechanics.

In early hominids it is known that Australopithecus afarensis shows some degree of axial orientation of molars in the expected direction (Ward et al., 1982). Other features of the dentition may be related: the distinct incurving of the maxillary arcade at $\mathrm{M}^{3}$ (see AL 233) is a feature that can be seen in other mammals that have moved the cheek teeth under the cranium. Notably, both Sciurus and Cebus (and recent Eskimos) show an incurved maxillary arcade, extreme axial tilts of third molars, and reduced third molars. All these should be signs of facial shortening or posterior positioning of the cheek teeth. The proportion of the dentition that is posterior to the zygomatic root has changed in human evolution. Chimpanzees (as a model of a protohominid) show the zygomatic root at $\mathrm{M}^{1} / \mathrm{M}^{2}$, and it is only at $\mathrm{M}^{3}$ that axial inclination becomes marked $\left(>5^{\circ}\right)$. In most humans this point is at $\mathrm{P}^{4} / \mathrm{M}^{1}$, and both $\mathrm{M}^{2}$ and $\mathrm{M}^{3}$ have fairly severe orientations. In Neanderthals more of the dentition is anterior to the zygomatic arch, and it is predicted that they should show milder molar inclinations than later Homo sapiens. Finally, axial inclinations of $20^{\circ}$ or more appear to be associated with extreme facial shortening and high levels of third molar agenesis.

These aspects of dental and cranial morphology deserve study separate from that of the complex determinants of the worn occlusal plane. Changes in axial orientation of molars should be closely related to evolutionary changes in the face and skull. Study of the functional determinants of molar axial inclination may contribute to a broader un. derstanding of patterns of mammalian craniofacial evolution. It is hoped that the preliminary proposals made here will be tested and clarified by future work.

\section{ACKNOWLEDGMENTS}

I thank the following individuals for their kind permission to study collections in their care: Professors P. Andrews at the British Museum of Natural History, Y. Coppens at the Musée de l'Homme, J.P. Garlick at Cambridge University, P.D. Gingerich and P. Meyers at the University of Michigan, J.L. Heim at l'Institut de Paléontologie $\mathrm{Hu}$ maine, C.F. Merbs and C.G. Turner II at Ar- izona State University, O.V. Nielsen at the University of Copenhagen, G.J. Romanes at the University of Edinburgh, E. Trinkaus at Harvard University, K.R. Turner at the University of Alabama, and D. Ubelaker at the Smithsonian Institution. I thank C.L. Brace, P.D. Gingerich, K.R. Rosenberg, and anonymous reviewers for comments that improved the manuscript. Karen Klitz drew the illustrations. This project was supported by grant BNS-7921733 from the National Science Foundation.

\section{LITERATURE CITED}

Ackerman, F (1953) Le Méchanisme des Machoires. Paris: Masson.

Bonwill, WGA (1899) The scientific articulation of the human teeth as founded on geometrical, mathematical and mechanical laws: The anatomical articulator. Dent. Items Interest 21:617-643, 873--880.

Campbell, TD (1925) Dentition and Palate of the Australian Aboriginal. Adelaide: The Hassell Press.

Clark, WE Le Gros (1955) The Fossil Evidence for Human Evolution. Chicago: University of Chicago Press.

Dempster, WT, Adams, WJ, and Duddles, RA (1963) Arrangement in the jaws of the roots of the teeth. J. Am. Dent. Assoc. 67:779-801.

Drennan, MR (1929) The dentition of a Bushman tribe. Ann. S. Afr. Mus. 24:61-87.

Hall, RL (1976) Functional relationships between dental attrition and the helicoidal plane. Am. J. Phys. Anthropol. 45:69-75.

Monson, GS (1920) Occlusion as applied to crown and bridgework. Am. J. Dent. Assoc. (J. Natl. Dent. Assoc.) $7: 399-413$.

Moses, CH (1946) Studies of wear, arrangement and occlusion of the dentitions of humans and animals and their relationship to orthodontia, periodontia, and prosthodontia. Dent. Items Interest 68:953-999.

Murphy, TR (1964) A biometric study of the helicoidal occlusal plane of the worn Australian dentition. Arch. Oral Biol. 9:255-267.

Oranje, $P$ (1934) The dentition of the Bush race. S. Afr. J. Sci. 31:576.

Osborn, JW (1982) Helicoidal plane of dental occlusion. Am. J. Phys. Anthropol. 57:273-282.

Pleasure, MA, and Friedman, SW (1938) Practical full denture occlusion. J. Am. Dent. Assoc. Dent. Cosmos 25:1606-1617.

Smith, BH (1983a) Dental Attrition in Hunter-Gatherers and Agriculturalists. Unpublished Ph.D. dissertation, The University of Michigan, Ann Arbor.

Smith, BH (1983b) Hominid attrition and occlusion at third molars: Understanding the helicoidal plane. Am. J. Phys. Anthropol. 60:255 (abstract).

Smith, BH (1984a) Patterns of molar wear in hunter gatherers and agriculturalists. Am. J. Phys. Anthropol. 63:39-56.

Smith, BH (1984b) Rates of molar wear: Implications for developmental timing and demography in human evolution. Am. J. Phys. Anthropol. 63:220 (abstract).

Spee, FG (1890) Die Verschiebungsbahn des Unterkiefers am Schädel. Arch. Anat. Physiol. 1890:285-294. 
Tobias, PV (1980) The natural history of the helicoidal occlusal plane and its evolution in early Homo. Am. J. Phys. Anthropol. 53:173-187.

Van Reenen, JF (1964) Dentition, jaws, and palate of the Kalahari Bushman (parts I-III). J. Dent. Assoc. S. Afr. 19:1-15, 38-44, 67-80.

Ward, SC (1981) The role of axial molar angulation in mediating helicoidal wear in Miocene apes, Pliocene hominids, and early Homo. Am. J. Phys. Anthropol. 54:288 (abstract).

Ward, SC, Johanson, DC, and Coppens, Y (1982) Subocclusal morphology and alveolar process relationships of hominid gnathic elements from the Hadar Formation: 1974-1977 collections. Am. J. Phys. Anthropol. 57:605-630. 